\title{
Primary Pulmonary Hypertension Associated with Asymptomatic Methylmalonic Aciduria in a Child
}

\author{
Fatma Zohra Chioukh and Kamel Monastiri
}

\begin{abstract}
Methylmalonic acidemia or aciduria (MMA) is an inborn error of metabolism that results in the accumulation of methylmalonic acid in blood with an increased excretion in urine. MMA usually presents in early infancy and its effects vary from mild to life-threatening. The clinical symptoms mainly include vomiting, dehydration, hypotonia, developmental delay, and failure to thrive. An association between pulmonary arterial hypertension (PAH) and MMA has been rarely reported. In the present work, the authors report a 16-month boy, who was admitted to the Pediatric Department for cyanosis and fever. He had a family history of primary pulmonary hypertension in a sister. The echocardiography showed a mild pericardial effusion and PAH. The metabolic screening led to the diagnosis of MMA. The condition of the baby worsened rapidly- and he died a few days later. Physicians should be aware about this atypical presentation of the disease, which can be fatal if not diagnosed and managed promptly.
\end{abstract}

Key Words: Metabolic disease, Methylmalonic aciduria, Pulmonary arterial hypertension.

\section{INTRODUCTION}

Pulmonary hypertension is a progressive and fatal disease. It may be due to pulmonary arterial hyper-tension (PAH), pulmonary venous hypertension, respiratory disorders and/or hypoxemia, thromboembolic diseases and disorders affecting directly the pulmonary vasculature. 1 An association with metabolic diseases was reported with Gaucher disease, type I, glycogen storage disease or pulmonary interstitial glycogenosis, nonketotic hyperglycinemia, mitochondrial disease and Cobalamin C defect, but rarely with methylmalonic acidemia (MMA). We report a new case of PAH associated with MMA in a child.

\section{CASE REPORT}

A 16-month boy, born at full-term, with weight $2.750 \mathrm{kgs}$, to first-degree relative parents, presented with a history of four episodes of bronchiolitis-like illness. The boy had a family history of an 18-month sister, who died of a primary pulmonary hypertension. He was admitted for fever $\left(38.7^{\circ} \mathrm{C}\right)$, coughing, cyanosis and tachycardia (200 beats $/ \mathrm{mn})$. He weighed $9 \mathrm{kgs}$ with delayed weight development. He had no dysmorphism or other clinical manifestation. A chest X-ray showed a globular heart with a cardiothoracic index of 0.58 and a mild right pleural effusion. Blood gases (pH: 7.48, PCo2: $3.67 \mathrm{kpa}$,

Department of Intensive Care and Neonatal Medicine, Faculty of Medicine, University of Monastir, Tunisia

Correspondence: Dr. Fatma Zohra Chioukh, Department of Intensive Care and Neonatal Medicine, Faculty of Medicine, Research Unit UR/01/08.14, University of Monastir, Tunisia E-mail: zohra_chioukh@yahoo.fr

Received: August 27, 2018; Accepted: February 19, 2019
PO2: $14.6 \mathrm{kpa}$, base excess (BE) -4.6, $\mathrm{HCO}_{3}: 17.8$ ) and blood glucose level were normal. Electrocardiogram showed sinus rhythm without any abnormalities of conduction. White blood count was $19,700 / \mathrm{mm}^{3}$, haemoglobin $(\mathrm{Hb}) 12 \mathrm{~g} / \mathrm{dl}$, platelets $362,000 / \mathrm{mm}^{3}$ and Creactive protein was $2.5 \mathrm{mg} / \mathrm{l}$. The echocardiography showed a mild pericardial effusion and $\mathrm{PAH}$. The baby was started on digoxin, captopril, and furosemid for PAH and aspirin for the treatment of pericarditis. The viral serologies for coxackie, adeno-virus, influenza, echovirus and infectious mononucleosis were negative. To investigate the $\mathrm{PAH}$, a metabolic screening was performed that showed on gas chromato-graphy mass spectroscopy (GC/MS) analysis of urine, the presence of markedly elevated levels of methyl-malonic acid $(1,580$ $\mu \mathrm{mol} / \mathrm{mmol}$ creatinine) and methyl citrate. Homocystein level was normal.

The condition of the baby worsened rapidly and a second echocardiography showed an enlarged right ventricle and an estimated systolic pulmonary arterial pressure (SPAP) of $90 \mathrm{mmHg}$. As prostin was not available, so sildenafil, represented an efficient alternative. Indeed, the right cardiac catheterisation showed a considerable decrease of SPAP $(-33 \%)$ with sildenafil. He was then treated with sildenafil $(0.5 \mathrm{mg} / \mathrm{kg} / 6 \mathrm{~h}$ orally), but his condition continued to worsen and he developed hepatomegaly, oedema and anaemia with $\mathrm{Hb}$ of $8 \mathrm{~g} / \mathrm{dl}$ and died, consequently. We were unable to precise the type of MMA.

\section{DISCUSSION}

PAH was clearly established in our patient. The diagnosis was made by a right-sided cardiac catheterisation, which is considered the gold standard confirmatory test. ${ }^{1}$ This PAH seemed to be familial, as the patient's sister died at the age of 18 months of primary PAH. 
This patient showed methylmalonic aciduria and apparently did not present with any of symptoms suggestive of the disease. However, the recurrent bronchiolitis-like episodes might be related to relapses of dyspnea secondary to metabolic acidosis. This finding was intriguing. Nevertheless, Leadly et al. reported asymptomatic children aged between 18 months and 13 years with methylmalonate accumulations in blood and urine and mutase deficiency was evidenced in all of them. ${ }^{2}$

An association between PAH and methylmalonic aciduria was first reported in a newborn in 2014. ${ }^{3}$ More recently, a 35-year female with MMA developed PAH 20 years after her first hemodialysis. However, although PAH may complicate regular hemodialysis, it was considered to be a complication of MMA, because it was responsive to medical treatment and reversible. 4 Other patients developed late-onset lung disease and PAH revealing Cobalamin $\mathrm{C}$ deficiency. ${ }^{5}$ The relationship between these two conditions is unclear, MMA could be a trigger enhancing PAH in cases of genetic or familial predisposition to PAH. PAH experts estimate that only $10 \%$ to $20 \%$ of the family members, that carry the gene, are responsible for $\mathrm{PAH}$ at risk to express the disease. 1

In this patient, it could be a pathogenic or a coincidental association between two distinct and rare diseases. PAH is associated with a number of other diseases or may develop after exposure to specific toxins and drugs; and MMA metabolites could be a trigger or a potential factor for the development of $\mathrm{PAH}$ in cases of genetic or familial predisposition to $\mathrm{PAH} .{ }^{1}$

Cullinane et al. reported pulmonary arterial media hyperplasia in two siblings with pulmonary hypertension and severe metabolic acidosis. ${ }^{6}$ Wideman et al. showed, by experimental studies on broilers, the influence of acute metabolic acidosis on pulmonary vascular resistance. 7 These reports could suggest a pathogenic association between MMA and PAH. Brandstetter et al. reported pulmonary vascular complications of MMA when associated with homocystinuria. 8 Liu et al. speculated that pulmonary microangiopathy, secondary to combined MMA and homocysteinemia, was the primary cause of diffuse lung disease with or without PAH. ${ }^{5}$ However, in all described MMA cases, such a complication has been rarely presented. This patient did not have such abnormalities and the clinical manifestations were different.

Sildenafil oral therapy was successfully tried in the treatment of PAH. ${ }^{9}$ In this case, it was proved efficient during right-side cardiac catheterisation. The fatal outcome of this patient in an anasarcous decompensation remains unexplained.

Taking into consideration our patient, we think that it is judicious, in case of primary $\mathrm{PAH}$, to search for MMA with or without homocysteinemia even when the patient has no suggestive signs. On the other hand, in case of "benign" MMA or classic MMA with cardio respiratory manifestations, arterial pulmonary pressure should be checked.

\section{REFERENCES}

1. Cheever $\mathrm{KH}$. An overview of pulmonary arterial hypertension: risks, pathogenesis, clinical manifestations, and management. J Cardiovasc Nurs 2005; 20:108-16.

2. Leadly FD, Levy HL, Shih VE, Benjamin R, Mahoney MJ. Benign methylmalonicaciduria. N Engl J Med 1984; 311:1015.

3. Agarwal R, Feldman GL, Poulik J, Stockton DW, Sood BG. Methylmalonicacidemia presenting as persistent pulmonary hypertension of the newborn. $J$ Neonatal Perinatal Med 2014; 7:247-51.

4. Kido J, Mitsubuchi H, Sakanashi M, Matsubara J, Matsumoto S, Sakamoto $\mathrm{R}$, et al. Pulmonary artery hypertension in methylmalonic acidemia. Hemodial Int 2017; 21:E25-9.

5. Liu J, Peng Y, Zhou N, Liu X, Meng Q, Xu H, et al. Combined methylmalonicacidemia and homocysteinuria presenting predominantly with late-onset diffuse lung disease: A case series of four patients. Orphanet J Rare Dis 2017; 12:58.

6. Cullinane C, Clarke JT, Rabinovich M, Bohn D, Silver MM. Hyperplasia of pulmonary arterial media in infantile familial pulmonary hypertension associated with severe metabolic acidosis. Mod Path 1993; 6:699-706.

7. Wideman RFJr, Kochera Kirby Y, Forman MF, Marson N, McNew RW, Owen RL. The infusion rate dependent influence of acute metabolic acidosis on pulmonary vascular resistance in broilers. Poult Sci 1998; 77:309-21.

8. Brandstetter Y, Weinhouse F, Splaingard ML, Tang TT. Cor pulmonale as a complication of methylmalonic acidemia and homocystinuria (Cbl-type). Am J Med Genet 1990; 36:167-71.

9. Humpl T, Rapes JT, Holtby H, Stephens D, Adatia I. Beneficial effect of oral sildenafil therapy on childhood pulmonary arterial hypertension: Twelve-month clinical trial, a single-drug, openlabel, pilot study. Circulation 2005; 111:3274-80. 